\title{
Endpoint estimates for first-order Riesz transforms associated to the Ornstein-Uhlenbeck operator
}

\author{
Giancarlo Mauceri, Stefano Meda and Peter Sjögren
}

\begin{abstract}
In the setting of Euclidean space with the Gaussian measure $\gamma$, we consider all first-order Riesz transforms associated to the infinitesimal generator of the Ornstein-Uhlenbeck semigroup. These operators are known to be bounded on $L^{p}(\gamma)$, for $1<p<\infty$. We determine which of them are bounded from $H^{1}(\gamma)$ to $L^{1}(\gamma)$ and from $L^{\infty}(\gamma)$ to $\operatorname{BMO}(\gamma)$. Here $H^{1}(\gamma)$ and $\operatorname{BMO}(\gamma)$ are the spaces introduced in this setting by the first two authors. Surprisingly, we find that the results depend on the dimension of the ambient space.
\end{abstract}

\section{Introduction}

Denote by $\gamma$ the normalised Gaussian measure on $\mathbb{R}^{d}$ having density $x \mapsto \pi^{-d / 2} \mathrm{e}^{-|x|^{2}}$ with respect to the Lebesgue measure. The Ornstein-Uhlenbeck operator $\mathcal{L}$ is the closure in $L^{2}(\gamma)$ of the operator $\mathcal{L}_{0}$, defined on $C_{c}^{\infty}\left(\mathbb{R}^{n}\right)$ by

$$
\mathcal{L}_{0}=-\frac{1}{2} \Delta+x \cdot \nabla
$$

where $\Delta$ and $\nabla$ denote the Euclidean Laplacian and gradient, respectively. The operator $\mathcal{L}$ is self-adjoint, and its spectral resolution is

$$
\mathcal{L}=\sum_{j=0}^{\infty} j \mathcal{P}_{j},
$$

where $\mathcal{P}_{j}$ denotes the orthogonal projection onto the linear span of Hermite polynomials of degree $j$ in $d$ variables. The Ornstein-Uhlenbeck operator generates a diffusion semigroup, which has been the object of many investigations during the last two decades. In particular, efforts have been made to study operators related 
to the Ornstein-Uhlenbeck semigroup, with emphasis on maximal operators (see [25], [12], [19], [10]), Riesz transforms (see [21], [13], [20], [23], [22], [14], [11], [5], $[6],[7],[24],[26],[4])$ and functional calculus ([8], [9], [17], [15]).

In this paper, we shall concentrate on the first-order Riesz transforms associated with $\mathcal{L}$. Since the eigenspace associated to the zero eigenvalue is nontrivial, the positive square root of $\mathcal{L}$ is not invertible, and one must define the Riesz transforms carefully. Consider the sequence $M: \mathbb{N} \rightarrow \mathbb{C}$, defined by

$$
M(j)= \begin{cases}0 & \text { if } j=0 \\ j^{-1 / 2} & \text { if } j=1,2, \ldots\end{cases}
$$

The operator $M(\mathcal{L})$, spectrally defined on $L^{2}(\gamma)$, extends to a bounded operator on $L^{p}(\gamma)$ for every $p$ in $(1, \infty)$ (see, for instance, Theorem 1.2 of [17]). The operator $M(\mathcal{L})$ plays, in this setting, the same role as the operator $(-\Delta)^{-1 / 2}$ in Euclidean harmonic analysis. We denote by $\partial_{i}$ the differentiation operator with respect to the variable $x_{i}$. The formal adjoint of $\partial_{i}$ in $L^{2}(\gamma)$ is the operator $\partial_{i}^{*}=2 x_{i}-\partial_{i}$. Notice that, at least formally,

$$
\sum_{i=1}^{d} \partial_{i}^{*} \partial_{i}=2 \mathcal{L}
$$

For each $i$ in $\{1, \ldots, d\}$, we define the operators $R_{i}, S_{i}, R_{i}^{*}$ and $S_{i}^{*}$ on finite linear combinations of Hermite polynomials by

$$
\begin{array}{ll}
R_{i}=\partial_{i} M(\mathcal{L}), & S_{i}=M(\mathcal{L}) \partial_{i}, \\
R_{i}^{*}=M(\mathcal{L}) \partial_{i}^{*}, & S_{i}^{*}=\partial_{i}^{*} M(\mathcal{L}) .
\end{array}
$$

It is straightforward to check that $R_{i}^{*}$ and $S_{i}^{*}$ are the formal adjoints in $L^{2}(\gamma)$ of $R_{i}$ and $S_{i}$, respectively. Recall the action of $\partial_{i}$ and $\partial_{i}^{*}$ on Hermite polynomials:

$$
\partial_{i} H_{n}=2 n H_{n-1} \quad \text { and } \quad \partial_{i}^{*} H_{n}=H_{n+1},
$$

where $H_{j}$ denotes the Hermite polynomials of degree $j$ in the $i^{\text {th }}$ variable. A straightforward argument, which uses these formulae and the expression for the $L^{2}(\gamma)$ norm of a Hermite polynomial, then shows that $R_{i}, R_{i}^{*}, S_{i}$ and $S_{i}^{*}$ extend to bounded operators on $L^{2}(\gamma)$, and that $R_{i}^{*}$ and $S_{i}^{*}$ are the Hilbert space adjoints of $R_{i}$ and $S_{i}$, respectively. Note that, in contrast with the Euclidean case, these transforms are not antisymmetric.

The operators $R_{i}, S_{i}, R_{i}^{*}$ and $S_{i}^{*}$ are all bounded on $L^{p}(\gamma)$ for each $p$ in $(1, \infty)$ (see [20]). This may be easily proved by using the commutation relations between $\partial_{i}$ and $\mathcal{L}$ and the spectral multiplier result (see Theorem 1.2 of [17]). It is straightforward to check that none of these operators are bounded on $L^{1}(\gamma)$ or on $L^{\infty}(\gamma)$. Weak type 1 estimates for $R_{i}$ and for $S_{i}^{*}$ have been proved in [5] and [1], respectively.

Thus, it is natural to ask what further estimates these operators satisfy at the endpoints $p=1$ and $p=\infty$. In the Euclidean case there are substitute results 
at $p=1$ and $p=\infty$ saying that the operators are bounded from $H^{1}\left(\mathbb{R}^{d}\right)$ to $L^{1}\left(\mathbb{R}^{d}\right)$ and from $L^{\infty}\left(\mathbb{R}^{d}\right)$ to $\operatorname{BMO}\left(\mathbb{R}^{d}\right)$. In our setting, the spaces $H^{1}(\gamma)$ and $\operatorname{BMO}(\gamma)$ were defined by Mauceri and Meda in [16], where these authors also developed a theory of singular integral operators in the Gaussian setting (see also [2] for a related theory in a more general setting). As applications, they proved that the imaginary powers of the Ornstein-Uhlenbeck operator are bounded from $H^{1}(\gamma)$ to $L^{1}(\gamma)$ and from $L^{\infty}(\gamma)$ to $\operatorname{BMO}(\gamma)$, and that the operators $R_{i}$ are bounded from $L^{\infty}(\gamma)$ to $\operatorname{BMO}(\gamma)$.

The question to be studied below is whether the Riesz transforms defined in (1.1) are bounded from $H^{1}(\gamma)$ to $L^{1}(\gamma)$ and from $L^{\infty}(\gamma)$ to $\operatorname{BMO}(\gamma)$. We find that this boundedness always holds in dimension one. But, surprisingly, in higher dimensions, each Riesz transform has one of these boundedness properties but not the other. Our results for $d \geq 2$ are summarised in the table in Theorem 3.1.

The paper is organised as follows. Section 2 contains background material and a few preliminary results, including the expressions for the kernels of $R_{i}$ and $S_{i}^{*}$. Our main result, Theorem 3.1, is stated in Section 3. Its proof constitutes Sections 4-7.

\section{Preliminaries}

We briefly recall the Hardy space $H^{1}(\gamma)$ from [16]. A Euclidean ball $B$ is called admissible if

$$
r_{B} \leq \min \left(1,1 /\left|c_{B}\right|\right)
$$

here and in the sequel $r_{B}$ and $c_{B}$ denote the radius and the centre of $B$, respectively. The collection of all admissible balls will be denoted by $\mathcal{B}_{1}$. When there is equality in (2.1), we say that $B$ is a maximal ball in $\mathcal{B}_{1}$.

A (Gaussian) atom is either the constant function 1 or a function $a$ in $L^{\infty}(\gamma)$, supported in an admissible ball $B$ and such that

$$
\|a\|_{\infty} \leq \gamma(B)^{-1} \text { and } \int_{\mathbb{R}^{n}} a \mathrm{~d} \gamma=0
$$

The space $H^{1}(\gamma)$ is then the vector space of all functions $f$ in $L^{1}(\gamma)$ that admit a decomposition of the form $\sum_{j} \lambda_{j} a_{j}$, where the $a_{j}$ are atoms and the sequence of complex numbers $\left\{\lambda_{j}\right\}$ is summable. The norm of $f$ in $H^{1}(\gamma)$ is defined as the infimum of $\sum_{j}\left|\lambda_{j}\right|$ over all representations of $f$ as above. In [16] the space $H^{1}(\gamma)$ is defined by means of $(1, p)$-atoms with $1<p<\infty$, but in [18] it is verified that the space obtained is the same as ours.

Note that $H^{1}(\gamma)$ is defined much as the atomic space $H^{1}$ on spaces of homogeneous type in the sense of R. R. Coifman and G. Weiss [3], but with one difference. Namely, only the exceptional atom and atoms with "small supports", i.e., with supports contained in admissible balls, appear in the definition of $H^{1}(\gamma)$. This difference is quite significant and has important consequences. 
It is known (Theorem 5.2 of [16]) that the Banach dual of $H^{1}(\gamma)$ is isomorphic to the space $\operatorname{BMO}(\gamma)$ of all functions of "bounded mean oscillation", i.e., of all functions in $L^{1}(\gamma)$ such that

$$
\|f\|_{*}=\sup _{B \in \mathcal{B}_{1}} \frac{1}{\gamma(B)} \int_{B}\left|f-f_{B}\right| \mathrm{d} \gamma<\infty,
$$

where $f_{B}=\frac{1}{\gamma(B)} \int_{B} f \mathrm{~d} \gamma$. A convenient norm on $\operatorname{BMO}(\gamma)$ is the following

$$
\|f\|_{\mathrm{BMO}}=\|f\|_{1}+\|f\|_{*} .
$$

Remark 2.1. An equivalent norm on $\operatorname{BMO}(\gamma)$ is obtained by replacing in $(2.3)$ balls in $\mathcal{B}_{1}$ by cubes of sidelength at most $2 \min \left(1,1 /\left|c_{B}\right|\right)$ (see Section 2 of [16]). As a consequence, a function $f$ is in $H^{1}(\gamma)$ if and only if it admits a decomposition of the form $\sum_{j} \lambda_{j} b_{j}$ where $\left\{\lambda_{j}\right\}$ is summable and the $b_{j}$ are either the constant function 1 or functions in $L^{\infty}(\gamma)$ supported in cubes $Q$ of sidelength at most $2 \min \left(1,1 /\left|c_{B}\right|\right)$, satisfying

$$
\|b\|_{\infty} \leq \gamma(Q)^{-1} \text { and } \quad \int_{\mathbb{R}^{n}} b \mathrm{~d} \gamma=0 .
$$

An equivalent norm on $H^{1}(\gamma)$ is then obtained by taking the infimum of $\sum_{j}\left|\lambda_{j}\right|$ over all representations of $f$ as above.

Given a bounded operator $T$ on $L^{2}(\gamma)$, we denote by $K_{T}$ the Schwartz kernel of $T$ and by $k_{T}$ the kernel of $T$ with respect to the Gaussian measure, defined by

$$
k_{T}(x, y)=\pi^{d / 2} \mathrm{e}^{|y|^{2}} K_{T}(x, y),
$$

in the sense of distributions in $\mathbb{R}^{d} \times \mathbb{R}^{d}$. The reason for introducing $k_{T}$ is that if $K_{T}$ is locally integrable in $\mathbb{R}^{d} \times \mathbb{R}^{d}$, then $T$ is an integral operator with kernel $k_{T}$ with respect to $\gamma$, i.e.,

$$
T f(x)=\int_{\mathbb{R}^{d}} k_{T}(x, y) f(y) \mathrm{d} \gamma(y) \quad \forall x \in \mathbb{R}^{d}, \quad \forall f \in C_{c}\left(\mathbb{R}^{d}\right) .
$$

One of the results in [16] says that if $T$ is bounded on $L^{2}(\gamma)$ and $k_{T}$ is locally integrable off the diagonal of $\mathbb{R}^{d} \times \mathbb{R}^{d}$, and satisfies

$$
\sup _{B \in \mathcal{B}_{1}} r_{B} \sup _{y \in B} \int_{(2 B)^{c}}\left|\nabla_{y} k_{T}(x, y)\right| \mathrm{d} \gamma(x)<\infty,
$$

then $T$ is bounded from $H^{1}(\gamma)$ to $L^{1}(\gamma)$, and, consequently, on $L^{p}(\gamma)$ for all $p$ in $(1,2)$. Notice that $(2.5)$ is a local Hörmander integral condition. Similarly, if

$$
\sup _{B \in \mathcal{B}_{1}} r_{B} \sup _{x \in B} \int_{(2 B)^{c}}\left|\nabla_{x} k_{T}(x, y)\right| \mathrm{d} \gamma(y)<\infty,
$$

then $T$ is bounded from $L^{\infty}(\gamma)$ to $\operatorname{BMO}(\gamma)$, and, consequently, on $L^{p}(\gamma)$ for all $p$ in $(2, \infty)$. 
We shall use these criteria to prove the boundedness results contained in Theorem 3.1, and start by determining the kernels of $R_{i}$ and of $S_{i}^{*}$. The functions $\phi$ and $\psi$, defined by

$$
\phi(r, x, y)=\frac{r y-x}{\sqrt{1-r^{2}}} \quad \text { and } \quad \psi(r, x, y)=\frac{r x-y}{\sqrt{1-r^{2}}}, \quad x, y \in \mathbb{R}^{d}, r \in(0,1),
$$

will occur frequently. Note that if $d \geq 2$, then $\phi$ and $\psi$ are vector-valued; their components will be denoted by $\phi_{i}$ and $\psi_{i}$. The arguments of these two functions will often be suppressed.

As shown in Lemma 2.2 of [7], the Schwartz kernel of the operator $M(\mathcal{L})$, defined in the introduction, is

$$
K_{M(\mathcal{L})}(x, y)=\pi^{-(d+1) / 2} \int_{0}^{1}\left[\frac{\mathrm{e}^{-|\psi|^{2}}}{\left(1-r^{2}\right)^{d / 2}}-\mathrm{e}^{-|y|^{2}}\right] \mathrm{d} \rho(r),
$$

where the measure $\rho$, supported in $[0,1]$, is defined by

$$
\mathrm{d} \rho(r)=\frac{\mathrm{d} r}{r \sqrt{-\log r}} .
$$

Hence the Schwartz kernel $K_{R_{i}}$ of the operator $R_{i}$ agrees off the diagonal with the function

$$
\partial_{x_{i}} K_{M(\mathcal{L})}(x, y)=-2 \pi^{-(d+1) / 2} \int_{0}^{1} \frac{r \psi_{i} \mathrm{e}^{-|\psi|^{2}}}{\left(1-r^{2}\right)^{(d+1) / 2}} \mathrm{~d} \rho(r) .
$$

By using (2.4) and the fact that $|\phi|^{2}-|\psi|^{2}=|x|^{2}-|y|^{2}$, we get that

$$
k_{R_{i}}(x, y)=-\frac{2}{\sqrt{\pi}} \mathrm{e}^{|x|^{2}} \int_{0}^{1} \frac{r \psi_{i} \mathrm{e}^{-|\phi|^{2}}}{\left(1-r^{2}\right)^{(d+1) / 2}} \mathrm{~d} \rho(r)
$$

for all $x \neq y$. To determine $k_{S_{i}^{*}}$, we argue similarly, observing that

$$
\partial_{x_{i}}^{*} \mathrm{e}^{-|\psi|^{2}}=\frac{-2 \phi_{i}}{\sqrt{1-r^{2}}} \mathrm{e}^{-|\psi|^{2}}
$$

and obtain

$$
\begin{aligned}
k_{S_{i}^{*}}(x, y) & =-\frac{2}{\sqrt{\pi}} \mathrm{e}^{|y|^{2}} \int_{0}^{1}\left[\frac{\phi_{i} \mathrm{e}^{-|\psi|^{2}}}{\left(1-r^{2}\right)^{(d+1) / 2}}+x_{i} \mathrm{e}^{-|y|^{2}}\right] \mathrm{d} \rho(r) \\
& =-\frac{2}{\sqrt{\pi}} \mathrm{e}^{|x|^{2}} \int_{0}^{1}\left[\frac{\phi_{i} \mathrm{e}^{-|\phi|^{2}}}{\left(1-r^{2}\right)^{(d+1) / 2}}+x_{i} \mathrm{e}^{-|x|^{2}}\right] \mathrm{d} \rho(r)
\end{aligned}
$$

for all $x \neq y$.

The constants $C<\infty$ and $c>0$ will depend only on the dimension $d$, and they may vary from occurrence to occurrence. By $f \sim g$ we mean $c<f / g<C$. Lebesgue measure is denoted $\lambda$. 


\section{Statements of results}

The main result of this paper is the following.

Theorem 3.1. In dimension one, the four operators $R_{1}, R_{1}^{*}, S_{1}$ and $S_{1}^{*}$ are bounded from $H^{1}(\gamma)$ to $L^{1}(\gamma)$ and from $L^{\infty}(\gamma)$ to $\operatorname{BMO}(\gamma)$.

In dimension $d \geq 2$, the boundedness properties of the operators $R_{i}, R_{i}^{*}, S_{i}$ and $S_{i}^{*}$ are given by the following table, where $B$ means "bounded" and $U$ "unbounded":

\begin{tabular}{|c||c|c|c|c|}
\hline & $R_{i}$ & $S_{i}$ & $R_{i}^{*}$ & $S_{i}^{*}$ \\
\hline \hline$H^{1} \rightarrow L^{1}$ & $U$ & $U$ & $B$ & $B$ \\
\hline$L^{\infty} \rightarrow \mathrm{BMO}$ & $B$ & $B$ & $U$ & $U$ \\
\hline
\end{tabular}

Remark 3.2. Note that all the operators $R_{i}$ and $S_{i}, i$ in $\{1, \ldots, d\}$, have the same boundedness properties, and so do the operators $R_{i}^{*}$ and $S_{i}^{*}$. Furthermore, the boundedness properties of $R_{i}^{*}$ and $S_{i}^{*}$ are "dual" to those of $R_{i}$ and $S_{i}$. This is not an accident, and the proofs of the statements concerning the boundedness properties of $R_{i}^{*}$ and $S_{i}$ will be obtained, by a duality argument, from the properties of $R_{i}$ and $S_{i}^{*}$.

In addition to $R_{i}, R_{i}^{*}, S_{i}$ and $S_{i}^{*}$ we also consider the operators $M_{i}$ and $M_{i}^{*}$, defined on Hermite polynomials by

$$
\begin{aligned}
M_{i} & =x_{i} M(\mathcal{L}), \\
M_{i}^{*} & =M(\mathcal{L}) x_{i} .
\end{aligned}
$$

Corollary 3.3. The operators $M_{i}$ and $M_{i}^{*}$ extend to bounded operators on $L^{p}(\gamma)$ for all $p$ in $(1, \infty)$ in any dimension. For $d=1$, they are bounded from $H^{1}(\gamma)$ to $L^{1}(\gamma)$ and from $L^{\infty}(\gamma)$ to $\operatorname{BMO}(\gamma)$. For $d \geq 2$, they are unbounded both from $H^{1}(\gamma)$ to $L^{1}(\gamma)$ and from $L^{\infty}(\gamma)$ to $\operatorname{BMO}(\gamma)$.

Proof. Since

$$
M_{i}=\frac{R_{i}+S_{i}^{*}}{2} \text { and } M_{i}^{*}=\frac{R_{i}^{*}+S_{i}}{2}
$$

the $L^{p}(\gamma)$ boundedness of $M_{i}$ and $M_{i}^{*}$ follows from that of $R_{i}, S_{i}, R_{i}^{*}$ and $S_{i}^{*}$ mentioned in the introduction. The remaining parts of the corollary are straightforward consequences of Theorem 3.1.

The proof of Theorem 3.1 occupies the remaining part of the paper. We first deal with $R_{i}$ and $S_{i}^{*}$. In Section 4 , it is proved that in dimension one, $R_{i}$ is bounded from $H^{1}(\gamma)$ to $L^{1}(\gamma)$ and $S_{i}^{*}$ from $L^{\infty}(\gamma)$ to $\operatorname{BMO}(\gamma)$. Section 5 deals with the positive statements concerning these two operators for $d \geq 2$, as indicated in the table. The negative results claimed for $R_{i}$ and $S_{i}^{*}$ are proved in Section 6. Finally, a rather simple duality argument in Section 7 gives all the results for the remaining operators $R_{i}^{*}$ and $S_{i}$. 


\section{Boundedness of $R_{1}$ and $S_{1}^{*}$ in one dimension}

Let $d=1$. We first prove that $R_{1}$ is bounded from $H^{1}(\gamma)$ to $L^{1}(\gamma)$. Because of (2.5) and (2.8), it suffices to show that there exists a constant $C$ such that for all balls $B$ in $\mathcal{B}_{1}$ and all points $y$ in $B$

$$
r_{B} \int_{(2 B)^{c}} \mathrm{~d} \lambda(x)\left|\int_{0}^{1} \frac{r \partial_{y}\left(\psi \mathrm{e}^{-\phi^{2}}\right)}{1-r^{2}} \mathrm{~d} \rho(r)\right| \leq C .
$$

Next, observe that

$$
\partial_{y}\left(\psi \mathrm{e}^{-\phi^{2}}\right)=-\frac{1}{\sqrt{1-r^{2}}} \mathrm{e}^{-\phi^{2}}-2 \phi \psi \frac{r}{\sqrt{1-r^{2}}} \mathrm{e}^{-\phi^{2}}=F_{1}(r, x, y)+F_{2}(r, x, y)
$$

To deal with $F_{2}$, we first note that $\partial \phi / \partial r=-\psi /\left(1-r^{2}\right)$, so that

$$
F_{2}(r, x, y)=-r \sqrt{1-r^{2}} \partial \mathrm{e}^{-\phi^{2}} / \partial r
$$

Integrating by parts, we get

$$
\int_{0}^{1} \frac{(-\log r)^{-1 / 2}}{1-r^{2}} F_{2}(r, x, y) \mathrm{d} r=\int_{0}^{1} \frac{\partial}{\partial r} \frac{r(-\log r)^{-1 / 2}}{\sqrt{1-r^{2}}} \mathrm{e}^{-\phi^{2}} \mathrm{~d} r .
$$

The integral to the right here is easily seen to be bounded by

$$
C \int_{0}^{1}(1-r)^{-2} \mathrm{e}^{-\phi^{2}} \mathrm{~d} r
$$

Considering also $F_{1}$, we can then estimate the expression in (4.1) by

$$
C r_{B} \int_{(2 B)^{c}} \int_{0}^{1}(1-r)^{-2} \mathrm{e}^{-\phi^{2}} \mathrm{~d} r \mathrm{~d} \lambda(x)
$$

Here we first integrate in $r$ only over $1-r_{B} /(2(1+|y|))<r<1$, which implies

$$
|r y-x|=|(r-1) y+y-x| \geq|y-x|-\frac{r_{B}|y|}{2(1+|y|)} \geq|y-x|-\frac{r_{B}}{2} \geq \frac{|y-x|}{2}
$$

and thus $|\phi| \geq|y-x| /(2 \sqrt{1-r})$. This part of (4.2) can thus be estimated by the following expression, where we make the change of variables $t=(1-r) /|x-y|^{2}$,

$$
\begin{aligned}
C r_{B} \int_{(2 B)^{c}} \int_{1-r_{B} /(2(1+|y|))}^{1} \frac{\mathrm{e}^{-c|x-y|^{2} /(1-r)}}{(1-r)^{2}} \mathrm{~d} r \mathrm{~d} \lambda(x) \leq \\
\leq C r_{B} \int_{(2 B)^{c}} \frac{\mathrm{d} \lambda(x)}{|x-y|^{2}} \int_{0}^{\infty} t^{-2} \mathrm{e}^{-c / t} \mathrm{~d} t \leq C .
\end{aligned}
$$


In the remaining part of (4.2), we switch the order of integration and find

$$
\begin{aligned}
r_{B} \int_{(2 B)^{c}} \mathrm{~d} \lambda(x) & \int_{0}^{1-r_{B} /(2(1+|y|))} \frac{\mathrm{e}^{-\phi(r, x, y)^{2}}}{(1-r)^{2}} \mathrm{~d} r \leq \\
& \leq r_{B} \int_{0}^{1-r_{B} /(2(1+|y|))} \frac{\mathrm{d} r}{(1-r)^{2}} \int_{\mathbb{R}^{d}} \mathrm{e}^{-\phi(r, x, y)^{2}} \mathrm{~d} \lambda(x) \\
& \leq C r_{B} \int_{0}^{1-r_{B} /(2(1+|y|))} \frac{\mathrm{d} r}{(1-r)^{3 / 2}},
\end{aligned}
$$

which is easily seen to be bounded by a constant independent of $y$ in $B$ and of $B$ in $\mathcal{B}_{1}$. The proof of the boundedness of $R_{1}$ from $H^{1}(\gamma)$ to $L^{1}(\gamma)$ is complete.

Now we prove that if $d=1$, then $S_{1}^{*}$ is bounded from $L^{\infty}(\gamma)$ to $\operatorname{BMO}(\gamma)$. Recall that the functions $\phi$ and $\psi$ are now scalar valued. By (2.6) and (2.9) it suffices to show that there exists a constant $C$, independent of $x$ in $B$ and of $B$ in $\mathcal{B}_{1}$, such that

$$
r_{B} \int_{(2 B)^{c}} \mathrm{~d} \lambda(y)\left|\int_{0}^{1} \frac{1}{1-r^{2}} \partial_{x}\left(\phi \mathrm{e}^{-\psi^{2}}+\left(1-r^{2}\right) x \mathrm{e}^{-y^{2}}\right) \mathrm{d} \rho(r)\right| \leq C .
$$

This is analogous to (4.1), with the following three differences. The variables $x$ and $y$, and thus also $\phi$ and $\psi$, are swapped; there is an extra factor $1 / r$; there is an extra term $\left(1-r^{2}\right) x \mathrm{e}^{-y^{2}}$.

For that part of the expression in (4.3) obtained by integrating only over $1 / 2<$ $r<1$, we can copy the argument used to verify (4.1) above. The extra factor $1 / r$ is harmless in this interval, and so is the term $\left(1-r^{2}\right) x \mathrm{e}^{-y^{2}}$. In the integration by parts with respect to $r$, one will now get an integrated term at $r=1 / 2$, equal to $C \mathrm{e}^{-\psi(1 / 2, x, y)^{2}}$ and easy to handle.

Consider then the integral over $0<r<1 / 2$. We have

$$
\begin{aligned}
\partial_{x}\left(\phi \mathrm{e}^{-\psi^{2}}\right. & \left.+\left(1-r^{2}\right) x \mathrm{e}^{-y^{2}}\right)= \\
& =-\left(1-r^{2}\right)\left[\frac{\mathrm{e}^{-\psi^{2}}}{\left(1-r^{2}\right)^{3 / 2}}-\mathrm{e}^{-y^{2}}\right]-\frac{2 \phi \psi r}{\left(1-r^{2}\right)^{1 / 2}} \mathrm{e}^{-\psi^{2}}
\end{aligned}
$$

Notice first that $r y-x=r(y-r x)+\left(r^{2}-1\right) x$, so that

$$
|\phi| \leq|\psi|+|x|
$$

This allows us to estimate the last term in (4.4) by $C r(1+|x|) \mathrm{e}^{-\psi^{2} / 2}$ for $0<r<1 / 2$. The corresponding part of the expression in (4.3) can then be seen not to be larger than $C r_{B}(1+|x|) \leq C$, since $x \in B$. For the first term in the right-hand side of (4.4), we write

$$
\frac{\mathrm{e}^{-\psi^{2}}}{\left(1-r^{2}\right)^{3 / 2}}-\mathrm{e}^{-y^{2}}=\frac{\mathrm{e}^{-\psi^{2}}-\mathrm{e}^{-y^{2}}}{\left(1-r^{2}\right)^{3 / 2}}+\left[\frac{1}{\left(1-r^{2}\right)^{3 / 2}}-1\right] \mathrm{e}^{-y^{2}} .
$$


The last term here is easily seen to be harmless and will be neglected. For the first term to the right, we observe that $\mathrm{e}^{-y^{2}}=\mathrm{e}^{-\psi(0, x, y)^{2}}$, so that

$$
\mathrm{e}^{-\psi(r, x, y)^{2}}-\mathrm{e}^{-y^{2}}=\int_{0}^{r} \frac{\partial}{\partial s} \mathrm{e}^{-\psi(s, x, y)^{2}} \mathrm{~d} s=\int_{0}^{r} \frac{2 \phi(s, x, y) \psi(s, x, y)}{1-s^{2}} \mathrm{e}^{-\psi(s, x, y)^{2}} \mathrm{~d} s .
$$

Since $s<r<1 / 2$ here, (4.5) implies that the last integral is at most

$$
C \int_{0}^{r}(1+|x|) \mathrm{e}^{-\psi(s, x, y)^{2} / 2} \mathrm{~d} s .
$$

Integrating this expression with respect to $y$, we get at most $C r(1+|x|)$. This will give a contribution to the expression in (4.3) which is at most $C r_{B}(1+|x|)$ $\int_{0}^{1 / 2}(-\log r)^{-1 / 2} \mathrm{~d} r$, and this is uniformly bounded with respect to $x$ in $B$ and $B$ in $\mathcal{B}_{1}$.

This concludes the proof that $S_{1}^{*}$ is bounded from $L^{\infty}(\gamma)$ to $\operatorname{BMO}(\gamma)$.

\section{Boundedness of $R_{i}$ and $S_{i}^{*}$ in higher dimensions}

The boundedness of $R_{i}$ from $L^{\infty}(\gamma)$ to $\operatorname{BMO}(\gamma)$ for any $d$ was proved in Theorem 7.2 (ii) of [16].

To prove that $S_{i}^{*}$ is bounded from $H^{1}(\gamma)$ to $L^{1}(\gamma)$ in all dimensions, we follow the lines of the proof of Theorem 7.2 in [16]. By (2.5) and (2.9), it is enough to verify that there exists a constant $C$, independent of $y$ in $B$ and of $B$ in $\mathcal{B}_{1}$ such that

$$
r_{B} \int_{(2 B)^{c}}\left|\int_{0}^{1} \nabla_{y}\left(\frac{\phi_{i} \mathrm{e}^{-|\phi|^{2}}}{\left(1-r^{2}\right)^{(d+1) / 2}}+x_{i} \mathrm{e}^{-|x|^{2}}\right) \mathrm{d} \rho(r)\right| \mathrm{d} \lambda(x) \leq C .
$$

It is straightforward to verify that all the components of the gradient with respect to $y$ that appear here are bounded in absolute value by

$$
C \frac{r\left(1+|\phi|^{2}\right)}{\left(1-r^{2}\right)^{(d+2) / 2}} \mathrm{e}^{-|\phi|^{2}} \leq C \frac{r}{\left(1-r^{2}\right)^{(d+2) / 2}} \mathrm{e}^{-|\phi|^{2} / 2} .
$$

Thus the left-hand side of (5.1) is no larger than

$$
C r_{B} \int_{(2 B)^{c}} \mathrm{~d} \lambda(x) \int_{0}^{1} \frac{r \mathrm{e}^{-|\phi|^{2} / 2}}{\left(1-r^{2}\right)^{(d+2) / 2}} \mathrm{~d} \rho(r) .
$$

We split the inner integral into integrals over $\left(0, r_{0}\right)$ and $\left(r_{0}, 1\right)$, with $r_{0}=$ $1-r_{B}^{2} / 4>1 / 2$. In each of the two resulting double integrals, we switch the order of integration. That part of (5.2) corresponding to $\left(0, r_{0}\right)$ will be less than

$$
\begin{aligned}
C r_{B} \int_{0}^{r_{0}} \frac{(-\log r)^{-1 / 2}}{\left(1-r^{2}\right)^{(d+2) / 2}} \mathrm{~d} r \int_{\mathbb{R}^{d}} \mathrm{e}^{-|\phi|^{2} / 2} \mathrm{~d} \lambda(x) & \leq C r_{B} \int_{0}^{r_{0}}(-\log r)^{-1 / 2}\left(1-r^{2}\right)^{-1} \mathrm{~d} r \\
& \leq C r_{B}\left(1-r_{0}\right)^{-1 / 2} \leq C
\end{aligned}
$$


To deal with the integral over $\left(r_{0}, 1\right)$, observe that $y \in B$ implies $|y| \leq c_{B}+$ $r_{B} \leq 2 r_{B}^{-1}$, in view of the definition of $\mathcal{B}_{1}$. For $x \notin 2 B$ and $r_{0}<r<1$ we then have

$$
|r y-x|=|y-x-(1-r) y|>r_{B}-|y| r_{B}^{2} / 4 \geq r_{B} / 2
$$

The remaining part of (5.2) is thus at most

$$
C r_{B} \int_{r_{0}}^{1} \frac{r}{\left(1-r^{2}\right)^{(d+2) / 2}} \mathrm{~d} \rho(r) \int_{|r y-x|>r_{B} / 2} \mathrm{e}^{-|\phi|^{2} / 2} \mathrm{~d} \lambda(x) .
$$

The inner integral in (5.3) is dominated by

$$
\left(1-r^{2}\right)^{d / 2} \int_{|z|>r_{B} /\left(2 \sqrt{1-r^{2}}\right)} \mathrm{e}^{-|z|^{2}} \mathrm{~d} z<C\left(1-r^{2}\right)^{d / 2}\left(r_{B} / \sqrt{1-r^{2}}\right)^{-2},
$$

and the whole expression (5.3) by

$$
C r_{B}^{-1} \int_{r_{0}}^{1}(1-r)^{-1 / 2} \mathrm{~d} r \leq C r_{B}^{-1}\left(1-r_{0}\right)^{1 / 2} \leq C .
$$

This concludes the proof of the boundedness of $S_{i}^{*}$ from $H^{1}(\gamma)$ to $L^{1}(\gamma)$.

\section{Unboundedness results}

In this section, we shall prove that if $d \geq 2$, then $R_{1}$ is unbounded from $H^{1}(\gamma)$ to $L^{1}(\gamma)$ and $S_{1}^{*}$ is unbounded from $L^{\infty}(\gamma)$ to $\operatorname{BMO}(\gamma)$. The proofs for $R_{2}, \ldots, R_{d}$ and $S_{2}^{*}, \ldots, S_{d}^{*}$ are similar and are omitted.

Consider first $R_{1}$. Let $Q$ be the cube with centre $(\xi, 0, \ldots, 0)$ and sidelength $2 \xi^{-1}$, for some large $\xi>0$. Denote by $Q_{+}$and $Q_{-}$those halves of $Q$ where $y_{2}>0$ and $y_{2}<0$, respectively. Set $b=\gamma(Q)^{-1}\left(\mathbf{1}_{Q_{+}}-\mathbf{1}_{Q_{-}}\right)$. By Remark 2.1, $\|b\|_{H^{1}(\gamma)}$ is bounded independently of $\xi$. Writing $\tilde{y}=\left(y_{1},-y_{2}, y_{3}, \ldots, y_{d}\right)$, one can easily see from (2.8) that

$$
\begin{aligned}
& \mathrm{e}^{-|x|^{2}} R_{1} a(x)= \\
& =\frac{2}{\sqrt{\pi} \gamma(Q)} \int_{Q_{+}} \int_{0}^{1} \frac{r\left(y_{1}-r x_{1}\right)}{\left(1-r^{2}\right)^{(d+2) / 2}}\left(\mathrm{e}^{-|\phi(r, x, y)|^{2}}-\mathrm{e}^{-|\phi(r, x, \tilde{y})|^{2}}\right) \mathrm{d} \rho(r) \mathrm{d} \gamma(y) \\
& =\frac{2}{\sqrt{\pi} \gamma(Q)} \int_{Q_{+}} \int_{0}^{1} \frac{r\left(y_{1}-r x_{1}\right)}{\left(1-r^{2}\right)^{(d+2) / 2}} \mathrm{e}^{-|\phi(r, x, y)|^{2}}\left(1-\mathrm{e}^{-\tau\left(r, x_{2}, y_{2}\right)}\right) \mathrm{d} \rho(r) \mathrm{d} \gamma(y)
\end{aligned}
$$

here and in the rest of the proof we write $\tau\left(r, x_{2}, y_{2}\right)$ in place of $4 r x_{2} y_{2} /\left(1-r^{2}\right)$. Similarly, we write $\eta\left(\xi, x_{1}\right)$ instead of $\sqrt{\left(\xi-x_{1}\right) / \xi}$. We shall evaluate $R_{1} a$ at points $x$ in the set

$$
\begin{aligned}
A_{Q}=\left\{x \in \mathbb{R}^{d}: x_{1} \in\left(\xi-1, \xi-\frac{4}{\xi}\right), x_{2} \in\left[\frac{\eta\left(\xi, x_{1}\right)}{2}, \eta\left(\xi, x_{1}\right)\right]\right. & \\
& \left.\left|x_{k}\right| \leq \eta\left(\xi, x_{1}\right), k=3, \ldots, d\right\} .
\end{aligned}
$$


Observe that if $x \in A_{Q}, y \in Q_{+}$and $r \in[0,1]$, then $y_{1}-r x_{1}>0$ and the integrands in the double integrals above are positive. With $\xi$ large and $x \in A_{Q}$, the integration in $r$ will be restricted to the interval

$$
I\left(x_{1}\right)=\left\{r \in(0,1):\left|r-\frac{x_{1}}{\xi}\right|<\frac{\eta\left(\xi, x_{1}\right)}{2 \xi}\right\} \subset(1 / 2,1) .
$$

For $x \in A_{Q}$ a lower bound for $\mathrm{e}^{-|x|^{2}} R_{1} a(x)$ is

$$
\frac{c}{\gamma(Q)} \int_{Q_{+}} \int_{I\left(x_{1}\right)} \frac{y_{1}-r x_{1}}{\left(1-r^{2}\right)^{(d+3) / 2}} \mathrm{e}^{-|\phi|^{2}}\left(1-\mathrm{e}^{-\tau\left(r, x_{2}, y_{2}\right)}\right) \mathrm{d} r \mathrm{~d} \gamma(y) .
$$

Therefore,

$$
\begin{aligned}
& \left\|R_{1} a\right\|_{1} \geq \int_{A_{Q}} R_{1} a(x) \mathrm{e}^{-|x|^{2}} \mathrm{~d} \lambda(x) \\
\geq & \frac{c}{\gamma(Q)} \int_{A_{Q}} \int_{Q_{+}} \int_{I\left(x_{1}\right)} \frac{y_{1}-r x_{1}}{\left(1-r^{2}\right)^{(d+3) / 2}} \mathrm{e}^{-|\phi|^{2}}\left(1-\mathrm{e}^{-\tau\left(r, x_{2}, y_{2}\right)}\right) \mathrm{d} r \mathrm{~d} \gamma(y) \mathrm{d} \lambda(x) .
\end{aligned}
$$

To prove that the operator $R_{1}$ is unbounded from $H^{1}(\gamma)$ to $L^{1}(\gamma)$, we only need to show that the last expression here tends to infinity with $\xi$. We claim that if $\xi$ is sufficiently large, one has for $x$ in $A_{Q}, y$ in $Q_{+}$and $r$ in $I\left(x_{1}\right)$

$$
\begin{aligned}
1-r^{2} & \sim \eta\left(\xi, x_{1}\right)^{2}, \\
y_{1}-r x_{1} & \geq c\left(\xi-x_{1}\right), \\
\mathrm{e}^{-|\phi(r, x, y)|^{2}} & \geq c, \\
1-\mathrm{e}^{-\tau\left(r, x_{2}, y_{2}\right)} & \sim y_{2} / \eta\left(\xi, x_{1}\right) .
\end{aligned}
$$

Deferring momentarily their proofs, we show how these inequalities yield the desired conclusion. Indeed, applying them and observing that

$$
\frac{1}{\gamma(Q)} \int_{Q_{+}} y_{2} \mathrm{~d} \gamma(y) \geq \frac{c}{\xi}
$$

we see from (6.2) that

$$
\begin{aligned}
\left\|R_{1} a\right\|_{1} & \geq \frac{c}{\gamma(Q)} \int_{A_{Q}} \int_{Q_{+}} \eta\left(\xi, x_{1}\right)^{-(d+2)} \xi y_{2}\left|I\left(x_{1}\right)\right| \mathrm{d} \gamma(y) \mathrm{d} \lambda(x) \\
& \geq c \int_{A_{Q}} \eta\left(\xi, x_{1}\right)^{-(d+1)} \xi^{-1} \mathrm{~d} \lambda(x)=c \int_{\xi-1}^{\xi-4 / \xi}\left(\xi-x_{1}\right)^{-1} \mathrm{~d} x_{1} \geq c \log \xi
\end{aligned}
$$

which tends to infinity with $\xi$ as desired.

It remains to prove the inequalities (6.3)-(6.6). Observe first that for $x \in A_{Q}$ one has $4 / \xi<\xi-x_{1}$. Considering also the geometric mean of these two quantities, we have

$$
4 / \xi<2 \eta\left(\xi, x_{1}\right)<\xi-x_{1}
$$


To verify (6.3), write for $r \in I\left(x_{1}\right)$

$$
1-r^{2} \sim 1-r=\eta\left(\xi, x_{1}\right)^{2}-\left(r-\frac{x_{1}}{\xi}\right) .
$$

Observe that $\left|r-x_{1} / \xi\right|<\eta\left(\xi, x_{1}\right)^{2} / 4$, because of the definition of $I\left(x_{1}\right)$ and (6.7), and (6.3) follows.

For (6.4), notice that similarly

$$
\begin{aligned}
y_{1}-r x_{1} & >\xi-\frac{1}{\xi}-\left(\frac{x_{1}}{\xi}+\frac{\xi-x_{1}}{4 \xi}\right) x_{1} \\
& >\xi-\frac{\xi-x_{1}}{4}-\frac{x_{1}^{2}}{\xi}-\frac{\xi-x_{1}}{4}>\frac{\xi^{2}-x_{1}^{2}}{\xi}-\frac{\xi-x_{1}}{2}>\frac{\xi-x_{1}}{2} .
\end{aligned}
$$

Aiming at (6.5), we write

$$
\begin{aligned}
\left|x_{1}-r y_{1}\right| & \leq\left|r\left(y_{1}-\xi\right)\right|+\left|\left(r-\frac{x_{1}}{\xi}\right) \xi\right| \\
& <\frac{1}{\xi}+\frac{1}{2 \xi} \eta\left(\xi, x_{1}\right) \xi<\eta\left(\xi, x_{1}\right) \leq C \sqrt{1-r^{2}},
\end{aligned}
$$

the last two inequalities in view of (6.7) and (6.3), respectively. Since $x \in A_{Q}$ and $y \in Q_{+}$, we similarly get for $k=2, \ldots, d$

$$
\left|x_{k}-r y_{k}\right| \leq\left|x_{k}\right|+\left|y_{k}\right| \leq \eta\left(\xi, x_{1}\right)+\xi^{-1} \leq C \sqrt{1-r^{2}}
$$

Thus $|x-r y|^{2} /\left(1-r^{2}\right) \leq C$, which implies (6.5).

Finally, we prove (6.6). Because of (6.3) and the facts that $x \in A_{Q}$ and $y \in Q_{+}$, one has

$$
\tau\left(r, x_{2}, y_{2}\right) \sim \frac{\xi}{\xi-x_{1}} \eta\left(\xi, x_{1}\right) y_{2}=\eta\left(\xi, x_{1}\right) y_{2}<\frac{1}{\sqrt{\xi\left(\xi-x_{1}\right)}}<1,
$$

and (6.6) follows.

This concludes the proof that $R_{1}$ is unbounded from $H^{1}(\gamma)$ to $L^{1}(\gamma)$.

Next, we prove that in higher dimension, $S_{1}^{*}$ is unbounded from $L^{\infty}(\gamma)$ to $\operatorname{BMO}(\gamma)$. We shall modify slightly the counterexample constructed above. The kernel is

$$
k_{S_{1}^{*}}(x, y)=\frac{2}{\sqrt{\pi}} \mathrm{e}^{|x|^{2}} \int_{0}^{1}\left[\frac{x_{1}-r y_{1}}{\left(1-r^{2}\right)^{(d+2) / 2}} \mathrm{e}^{-|\phi|^{2}}+x_{1} \mathrm{e}^{-|x|^{2}}\right] \mathrm{d} \rho(r) .
$$

With $\xi>0$ large, we let the cube $Q$, its subset $Q_{+}$and the region $A_{Q}$ be as above. But now $f$ will be the characteristic function of $A_{Q}$, and $S_{1}^{*} f$ will be evaluated in $Q$. For $x \in Q_{+}$we write $\tilde{x}=\left(x_{1},-x_{2}, x_{3}, \ldots, x_{d}\right)$. We shall verify that

$$
\frac{1}{\gamma(Q)} \int_{Q_{+}}\left(S_{1}^{*} f(x)-S_{1}^{*} f(\tilde{x})\right) \mathrm{d} \gamma(x)
$$


tends to $+\infty$ as $\xi$ tends to $+\infty$. This would clearly mean that $\left\|S_{1}^{*} f\right\|_{\text {BMO }}$ also tends to infinity, although $\|f\|_{\infty}=1$ for all $\xi$. Notice that for $x$ in $Q_{+}$

$$
\begin{aligned}
& S_{1}^{*} f(x)-S_{1}^{*} f(\tilde{x})= \\
& =\frac{2}{\sqrt{\pi}} \int_{A_{Q}} \int_{0}^{1} \frac{x_{1}-r y_{1}}{\left(1-r^{2}\right)^{(d+3) / 2}} \mathrm{e}^{-|\psi|^{2}}\left(1-\mathrm{e}^{-\tau\left(r, x_{2}, y_{2}\right)}\right) \mathrm{d} \rho(r) \mathrm{d} \lambda(y)
\end{aligned}
$$

recall that we write $\tau\left(r, x_{2}, y_{2}\right)$ in place of $4 r x_{2} y_{2} /\left(1-r^{2}\right)$. Here the integrand is positive. We now integrate with respect to $\mathrm{d} \gamma(x)$ over $x \in Q_{+}$, and restrict the integral in $r$ to the interval $I\left(y_{1}\right) \subset(1 / 2,1)$, defined like $I\left(x_{1}\right)$ (see (6.1)). For such $r$, one can replace the measure $\mathrm{d} \rho(r)$ by $\left(1-r^{2}\right)^{-1 / 2} \mathrm{~d} r$. This leads to a lower estimate of the quantity (6.8) in terms of a triple integral rather similar to that in (6.2). The only differences are that $x$ and $y$ have switched roles and that we now have also an insignificant factor $1 / r$. Thus the integral will tend to $\infty$ with $\xi$, like that in $(6.2)$.

This concludes the proof that $S_{1}^{*}$ is unbounded from $L^{\infty}(\gamma)$ to $\operatorname{BMO}(\gamma)$.

\section{Duality arguments}

All the statements contained in Theorem 3.1 that have not been proved yet will follow from those proved above by a simple duality argument, based on the following elementary lemma. We point out that by a result of [18], $L^{2}(\gamma)$ is a subspace of $H^{1}(\gamma)$, which is dense because it contains all the atoms.

Lemma 7.1. Suppose that $T$ is a bounded operator on $L^{2}(\gamma)$. Then $T$ extends to a bounded operator from $H^{1}(\gamma)$ to $L^{1}(\gamma)$ if and only if its (Hilbert space) adjoint operator $T^{*}$ is bounded from $L^{\infty}(\gamma)$ to $\operatorname{BMO}(\gamma)$.

Proof. Suppose that $T$ extends to a bounded operator from $H^{1}(\gamma)$ to $L^{1}(\gamma)$. Then for $g$ in $L^{\infty}(\gamma)$

$$
\left\|T^{*} g\right\|_{\mathrm{BMO}}=\sup \left\{\left|\left\langle f, T^{*} g\right\rangle\right|: f \in L^{2}(\gamma),\|f\|_{H^{1}} \leq 1\right\},
$$

and

$$
\left|\left\langle f, T^{*} g\right\rangle\right|=|\langle T f, g\rangle| \leq\|T f\|_{1}\|g\|_{\infty} \leq\|T\|_{H^{1}, L^{1}}\|f\|_{H^{1}}\|g\|_{\infty} .
$$

Thus $T^{*}$ is bounded from $L^{\infty}(\gamma)$ to $\operatorname{BMO}(\gamma)$.

The converse implication is proved similarly. We omit the details.

Now, the boundedness in one dimension of $R_{1}^{*}$ from $L^{\infty}(\gamma)$ to $\operatorname{BMO}(\gamma)$ follows by Lemma 7.1 from that of $R_{1}$ from $H^{1}(\gamma)$ to $L^{1}(\gamma)$, proved in Section 4. Similarly, the unboundedness in dimension $d \geq 2$ of $R_{i}^{*}$ from $L^{\infty}(\gamma)$ to $\operatorname{BMO}(\gamma)$ follows from the unboundedness of $R_{i}$ from $H^{1}(\gamma)$ to $L^{1}(\gamma)$ proved in Section 6 , and the boundedness of $R_{i}^{*}$ from $H^{1}(\gamma)$ to $L^{1}(\gamma)$ for any $d$ follows from that of $R_{i}$ from $L^{\infty}(\gamma)$ to $\operatorname{BMO}(\gamma)$ proved in Section 5 . 
A similar argument gives the boundedness of $S_{i}$ from $H^{1}(\gamma)$ to $L^{1}(\gamma)$ when $d=1$, that of $S_{i}$ from $L^{\infty}(\gamma)$ to $\operatorname{BMO}(\gamma)$ for any $d$, and the unboundednes of $S_{i}$ from $H^{1}(\gamma)$ to $L^{1}(\gamma)$ when $d \geq 2$.

The theorem is completely proved.

\section{References}

[1] Aimar, H., Forzani, L. And Scotto, R.: On Riesz transforms and maximal functions in the context of Gaussian harmonic analysis. Trans. Amer. Math. Soc. 359 (2007), no. 5, 2137-2154.

[2] Carbonaro, A., Mauceri, G. and Meda, S.: $H^{1}$ and BMO for certain locally doubling metric measure spaces of finite measure. Colloq. Math. 118 (2010), no. 1, $13-41$.

[3] Coifman, R. R. And Weiss, G.: Extensions of Hardy spaces and their use in analysis. Bull. Amer. Math. Soc. 83 (1977), no. 4, 569-645.

[4] Dragicevic, O. and Volberg, A.: Bellman functions and dimensionless estimates of Littlewood-Paley type. J. Operator Theory 56 (2006), no. 1, 167-198.

[5] Fabes, E. B., Gutiérrez, C. And Scotto, R.: Weak type estimates for the Riesz transforms associated with the Gaussian measure. Rev. Mat. Iberoamericana 10 (1994), no. 2, 229-281.

[6] Forzani, L. And Scotto, R.: The higher order Riesz transforms for Gaussian measure need not be weak type (1,1). Studia Math. 131 (1998), no. 3, 205-214.

[7] García-Cuerva, J., Mauceri, G. and Sjögren, P. and Torrea, J. L.: Higherorder Riesz operators for the Ornstein-Uhlenbeck semigroup. Potential Anal. 10 (1999), no. 4, 379-407.

[8] García-Cuerva, J., Mauceri, G., Sjögren, P. and Torrea, J. L.: Spectral multipliers for the Ornstein-Uhlenbeck semigroup. J. Anal. Math. 78 (1999), 281-305.

[9] García-Cuerva, J., Mauceri, G., Meda, S., Sjögren, P. and Torrea, J. L.: Functional calculus for the Ornstein-Uhlenbeck Operator. J. Funct. Anal. 183 (2001), no. 2, 413-450.

[10] García-Cuerva, J., Mauceri, G., Meda, S., Sjögren, P. and Torrea, J. L.: Maximal operators for the holomorphic Ornstein-Uhlenbeck semigroup. J. London Math. Soc. (2) 67 (2003), no. 1, 219-234.

[11] Gutiérrez, C. E., Segovia, C. And Torrea, J. L.: On higher Riesz transforms for Gaussian measures. J. Fourier. Anal. Appl. 2 (1996), no. 6, 583-596.

[12] Gutiérrez, C. E. And Urbina, W.: Estimates for the maximal operator of the Ornstein-Uhlenbeck semigroup. Proc. Amer. Math. Soc. 113 (1991), no. 1, 99-104.

[13] Gundy, R.F.: Sur les transformations de Riesz pour le semi-groupe d'OrnsteinUhlenbeck. C. R. Acad. Sci. Paris Sci. Sér. I Math. 303 (1986), no. 19, 967-970.

[14] Gutiérrez, C. E.: On the Riesz transforms for Gaussian measures. J. Funct. Anal. 120 (1994), no. 1, 107-134.

[15] Hebisch, W., Mauceri, G. And Meda, S.: Holomorphy of spectral multipliers of the Ornstein-Uhlenbeck operator. J. Funct. Anal. 210 (2004), no. 1, 101-124. 
[16] Mauceri, G. And Meda, S.: BMO and $H^{1}$ for the Ornstein-Uhlenbeck operator. J. Funct. Anal. 252 (2007), no. 1, 278-313.

[17] Mauceri, G., Meda, S. And Sjögren, P.: Sharp estimates for the OrnsteinUhlenbeck operator. Ann. Sc. Norm. Super. Pisa Cl. Sci. (5) 3 (2004), no. 3, 447-480.

[18] Mauceri, G., Meda, S. And Sjögren, P.: A maximal function characterisation of the Hardy space for the Gauss measure. arXiv: 1006.5551. To appear in Proc. Amer. Math. Soc.

[19] Menárguez, T., PÉrez, S. And Soria, F.: The Mehler maximal function: a geometric proof of the weak type 1. J. London Math. Soc. (2) 61 (2000), no. 3, 846-856.

[20] Meyer, P. A.: Transformations de Riesz pour les lois Gaussiennes. In Seminar on probability, XVIII, 179-193. Lecture Notes in Math. 1059, Springer, Berlin, 1984.

[21] Muckenhoupt, B.: Hermite conjugate expansions. Trans. Amer. Math. Soc. 139 (1969), 243-260.

[22] PÉRez, S.: The local part and the strong type for operators related to the Gaussian measure. J. Geom. Anal. 11 (2001), no. 3, 491-507.

[23] PIsier, G.: Riesz transforms: a simpler analytic proof of P. A. Meyer's inequality. In Séminaire de probabilités, XXII, 485-501. Lecture Notes in Math. 1321, Springer, Berlin, 1988.

[24] PÉRez, S. And Soria, F.: Operators associated with the Ornstein-Uhlenbeck semigroup. J. London Math. Soc. (2) 61 (2000), no. 3, 857-871.

[25] Sjögren, P.: On the maximal function for the Mehler kernel. In Harmonic analysis (Cortona, 1982), 73-82. Lecture Notes in Math. 992, Springer, Berlin, 1983.

[26] Urbina, W.: On singular integrals with respect to the Gaussian measure. Ann. Scuola Norm. Sup. Pisa Cl. Sci. (4) 17 (1990), no. 4, 531-567.

Received January 17, 2010.

Giancarlo Mauceri: Dipartimento di Matematica, Università di Genova, via Dodecaneso 35, 16146 Genova, Italia.

E-mail: mauceri@dima.unige.it

Stefano Meda: Dipartimento di Matematica e Applicazioni, Università di MilanoBicocca, via R. Cozzi 53, 20125 Milano, Italy.

E-mail: stefano.meda@unimib.it

Peter Sjögren: Mathematical Sciences, University of Gothenburg and Mathematical Sciences, Chalmers, 41296 Göteborg, Sweden.

E-mail: peters@chalmers.se

Work partially supported by the Progetto Cofinanziato "Analisi Armonica". 